Classification

Physics Abstracts

$05.40-64.60-02.50$

\title{
A random fuse model for breaking processes
}

\author{
L. de Arcangelis, S. Redner \\ Center for Polymer Studies and Department of Physics (*), \\ Boston University, Boston, MA 02215, U.S.A.
}

and H. J. Herrmann

SPT, CEN Saclay, 91191 Gif sur Yvette Cedex, France

(Reçu le 11 avril 1985, accepté le 14 mai 1985)

\begin{abstract}
Résumé. - Nous introduisons et étudions un modèle simple pour décrire les phénomènes de rupture. Ce modèle est défini par un réseau aléatoire d'isolants et de fusibles. Les propriétés de conductivité électrique sont analysées : en augmentant la tension électrique appliquée, une séquence de fusibles va sauter et se transformer d'une manière irréversible en isolants. Le processus se termine dès qu'il n'y a plus de chemin conducteur qui traverse le réseau. Des quantités comme la conductivité totale du réseau et la tension appliquée de l'extérieur sont calculées pendant la rupture. Deux nouveaux exposants critiques qui décrivent ces quantités près du seuil de percolation ont été déterminés.

Abstract. - We introduce and study a simple idealized model to describe breaking processes by analysing the current-carrying properties of a random network consisting of insulators and fuses. By increasing the value of the external voltage applied across the network, a sequence of fuses will " burn out " and change irreversible into insultating bonds. This process terminates when a conducting path no longer exists in the network. We monitor several basic quantities during this breaking process, such as the conductivity of the network and the value of the voltage needed to break the hottest fuse. Two new exponents describing the behaviour of these quantities near the percolation threshold are reported.
\end{abstract}

Breaking phenomena of disordered structures such as the tearing of unwoven textiles, the fracture of brittle materials, or the propagation of cracks in solids are receiving increasing attention due to their imminent technological relevance and due to the fundamental theoretical questions involved [1-7]. The irreversibility and non-locality of breaking processes produces rather complicated spatial and temporal correlations, giving rise to a potentially much richer phenomenology than the extensively studied steady-state properties of disordered media, such as the electrical conductivity or superconductivity [8-11], or the linear elasticity of random networks [12-14]. There is a vast literature on the general problem of mechanical failure and crack propagation in solids $[1,6,7]$. Unfortunately much of the existing work involves very complicated models and/or calculations. Our motivation is to introduce a relatively simple and tractable model which captures at least some of the basic features of breaking processes. To this end, we consider the electrical analogue of breaking through the introduction of a random fuse network.

(*) Supported in part by grants from the ARO, NSF, and ONR. 
The " burning out " of individual fuses as the external voltage is increased is analogous to the physical breaking of bonds as the mechanical stress across an elastic network is increased. One of the motivations for dealing with such an electrical network is that it is the scalar analogue of an elasticity problem, and the former should therefore be simpler to study. This is similar to the situation in the linear response regime, where the scalar conductivity problem is much simpler to treat than the vector elasticity problem.

In our model, we consider a square lattice of size $L \times L$ with bonds all of length unity and free boundary conditions. On each bond of this lattice, we place a fuse with probability $p$ and an insulator with probability $1-p$. We define a fuse as a device with a constant resistance when the applied voltage across it is less than a critical value $V_{c}$ (Fig. 1). For convenience we choose the value of the fuse resistance and the value of $V_{c}$ to be unity. If the applied voltage exceeds $V_{c}$, the fuse burns out or " breaks » irreversibly, thus changing into an insulator. We are interested in studying how a random network consisting of these fuses will break apart, i.e., undergo an insulating transition, as the external voltage across the system is increased.

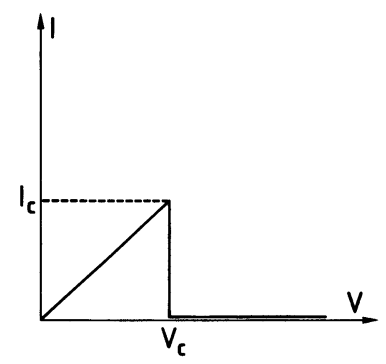

Fig. 1. - The current-voltage response of the fuse element used in our model network.

To study this breaking process, we first identify the conducting backbone of the network, defined as that portion of the system which conducts current, by the use of the recently introduced cluster « burning " algorithm [15]. On this backbone, we then calculate the voltages at each node of the network by using a standard Gauss-Seidel relaxation procedure. In this set of voltages, there will exist a " hottest » bond across which the potential drop has the largest value. We now adjust the value of the external voltage so that the voltage drop across the hottest bond is just sufficient to cause its breaking. In order to obtain an intrinsic quantity, we normalize this critical voltage by the length of the system. We term the value of the external voltage obtained by this procedure as $V_{\text {in }}$, the " initial " value of the voltage required to initiate the breaking process. In performing subsequent breakings, we first assume that there is sufficient time for the modified network, in which the hottest bond has been removed, to reach a new equilibrium voltage state before further fuse burning takes place. In the framework of our model, we therefore recalculate the new equilibrium values of the node voltages when the hottest bond has been deleted. Now a new " hottest " bond may be identified, and then broken. This process is repeated until the network is broken into two distinct pieces (Fig. 2).

The breaking process that we have defined is somewhat different than what might be realized in a simple-minded experimental procedure. A typical procedure would be to increase the value of the external voltage, breaking bonds one at a time until the entire network breaks apart. For a relatively homogeneous network with $p \cong 1$, the breaking process would be self-sustaining as soon as the first bond is broken due to the propagation of a well-defined crack. On the other hand, for a network with $p \simeq p_{c}$, the percolation threshold, it may be necessary to increase the voltage several times after the first bond breaking, as there may exist very weak strands which break initially, but other stronger strands may require a still higher value of the voltage to break.

For the homogeneous network, the propagation of a crack causes the system to weaken mono- 


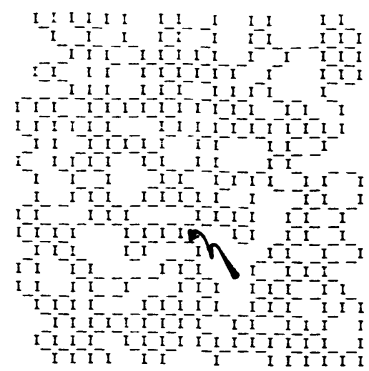

(a)

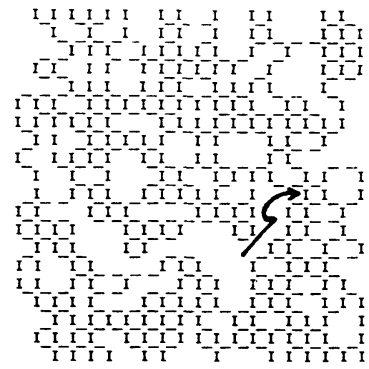

(c)

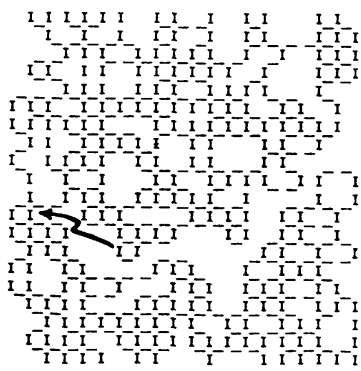

(e)

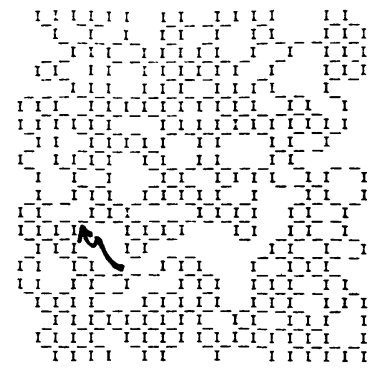

(b)

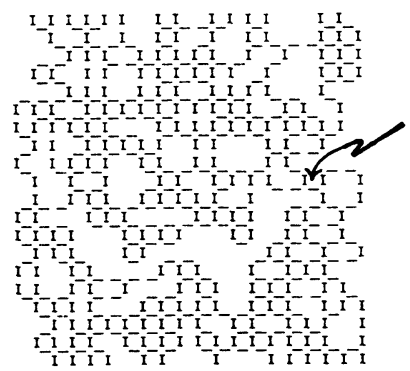

(d)

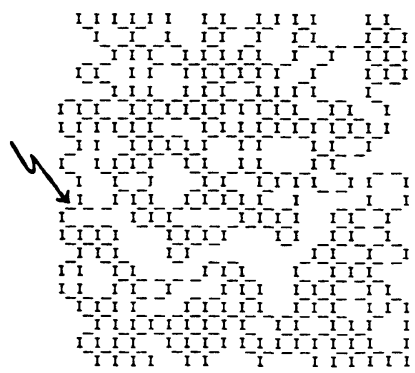

(f)

Fig. 2. - Illustration of a typical breaking process for a $20 \times 20$ square lattice network at $p=0.7$ (a-f). Only the conducting backbone of the full random network is shown. For each network, we indicate the hottest bond in the network by the arrow. The values of the external voltage needed to break the hottest bond are, respectively : (a) 0.0985 , (b) 0.102 , (c) 0.0964 , (d) 0.118 , (e) 0.0981 , and (f) 0.113 . Open boundary conditions are used in horizontal direction and fixed boundary conditions in vertical direction.

tonically. Due to this weakening, the value of the external voltage could be decreased to just above the breaking voltage of the most highly-stressed intact bond without interrupting the overall breaking process. Our numerical procedure of breaking only one bond (the hottest) at a time corresponds to adjusting the external voltage upward or possibly downward after each fuse burning, so that the voltage drop across the hottest bond in the network just exceeds its breaking point. For a homogeneous network, this numerical approach should give results similar to that obtained in the naive experimental procedure that we have outlined above. However, near the percolation threshold, there may be some differences in the results obtained by the two methods.

Just before the network breaks, we reach a rather singular situation in which the conductivity of the network is limited by a single bond along which the entire current of the network must 
pass through. We term the value of the external voltage (per unit length of the system) required to break this final bond as $V_{\text {fin }}$. The critical behaviour of both $V_{\text {in }}$ and $V_{\text {fin }}$ as a function of $p-p_{\text {c }}$ appears to be rather interesting, as shown in figure 3. At $p=1, V_{\text {fin }}$ equals unity as the potential drop across each bond of the network is the same. Then as $p$ decreases from unity, $V_{\text {in }}$ decreases as well, since the network is becoming " weaker " in a homogeneous way. However, for $p \simeq 0.7$, a further decrease in $p$ leads to a subsequent increase in $V_{\text {in }}$. The origin of this increase stems from the fact that near $p_{\mathrm{c}}$, the conducting paths become increasingly longer and more tortuous. In order to achieve the critical potential drop of unity across one of the bonds in such a path, it will be necessary to have a relatively large value of the external voltage.

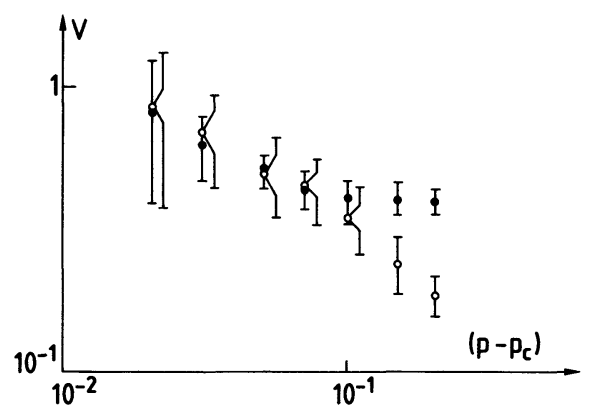

Fig. 3. - Double logarithmic plot of the critical value of the external voltage (per unit system length) defined as the voltage required to burn the next fuse, versus $p-p_{\mathrm{c}}$ for an $80 \times 80$ square lattice network. Shown is the voltage, $V_{\text {in }}$, before any fuse is burned ( $(\bullet)$, and $V_{\text {fin }}$, the voltage just before the last fuse is burned (O). Statistics of 25 for $p>0.55$ and of 50 for $p \leqslant 0.55$.

On the other hand, $V_{\text {fin }}$ exhibits a monotonic dependence on $p$. For $p$ close to unity, it is clear that $V_{\text {fin }}$ will be considerably smaller than $V_{\text {in }}$. Numerically we find that for a network at $p=1$, the ratio $V_{\text {fin }} / V_{\text {in }}$ appears to approach a finite value as the linear dimension of the network becomes large. As $p \rightarrow p_{c}$, the number of bonds that need to be cut in order to disconnect the network approaches unity, so that $V_{\text {in }}$ and $V_{\text {fin }}$ should coincide, and this is what we observe from our data. The behaviour of $V_{\text {fin }}$ as $p \rightarrow p_{\mathrm{c}}$ is consistent with a power law divergence, $V_{\text {fin }} \simeq\left(p-p_{\mathrm{c}}\right)^{-z}$, with $z=0.48 \pm 0.08$, a new exponent which seems to be unrelated to any of the known exponents of the percolation problem.

In addition to studying the value of the voltage required to cause breaking, we have found that two additional quantities appear to exhibit simple power-law dependences on $p-p_{\mathrm{c}}$. The first quantity is the average number of bonds per unit volume, $\langle N\rangle$, which must be cut in order to disconnect the network. Appealing to the nodes and links picture of the percolating network [16-17], we expect that the network will become disconnected when an entire row of links is broken. Therefore we expect that $\langle N\rangle$ should vary inversely with the correlation length, $\xi$. In our simulations of an $80 \times 80$ network (Fig. 4), the data suggest that $\langle N\rangle$ vanishes near $p_{\mathrm{c}}$ as $\left(p-p_{c}\right)^{x}$, with $x=1.40 \pm 0.15$, in reasonable agreement with the hypothesis that $\langle N\rangle$ should be proportional to the inverse correlation length.

A final quantity that we monitor is the conductivity of the network during the breaking process. The value of the network conductivity before any breaking occurs, coincides with the conductivity of the classical random resistor network problem. We define the value of the conductivity of this initial system as $G_{\text {in }}$. The value of $G_{\text {in }}$ should vanish near $p_{\mathrm{c}}$ as $\left(p-p_{\mathrm{c}}\right)^{t}$, with $t=1.28$ [8-11]. Our data on a $80 \times 80$ lattice gives a value for $t$ of $1.18 \pm 0.15$, thereby yielding an estimate of the accuracy of our calculations. Just before the network completely breaks, we also measure the conductivity of the resulting " final » network, and we define the measured value of the conductivity to be $G_{\mathrm{fin}}$. This quantity appears to vanish near $p_{\mathrm{c}}$ as $\left(p-p_{\mathrm{c}}\right)^{v}$, with $v=0.53 \pm 0.10$. 


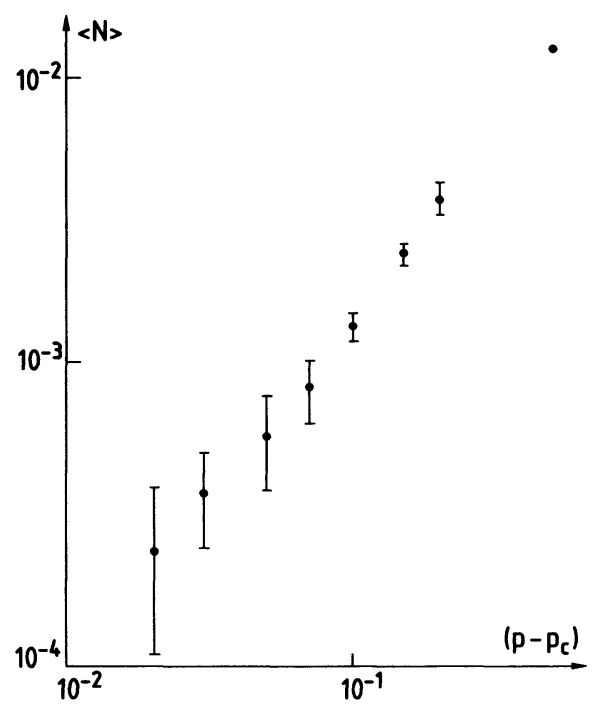

Fig. 4. - Double logarithmic plot of the average number of burned fuses per unit volume, $\langle N\rangle$, as a function of $p-p_{\mathrm{c}}$, for the $80 \times 80$ network. The error bars indicate the statistical uncertainties. Statistics as in figure 3 .

There appears to be no simple connection between this new exponent and the other known exponents of percolation (Fig. 5).

It is also interesting to note that $G_{\text {fin }}$ possesses a peculiar scaling property. At $p_{\mathrm{c}}$, only one bond must be broken in order to render the network disconnected [18], so that $G_{\text {in }}$ and $G_{\text {fin }}$ must coincide. This coincidence will persist for values of $p$ greater than $p_{\mathrm{c}}$ as long as $\xi$ is larger than the linear dimension, $L$, of the system under study. However, once $\xi$ becomes smaller than $L$, many bond breakings are required to disconnect the network and $G_{\text {fin }}$ will now be smaller than $G_{\text {in }}$.

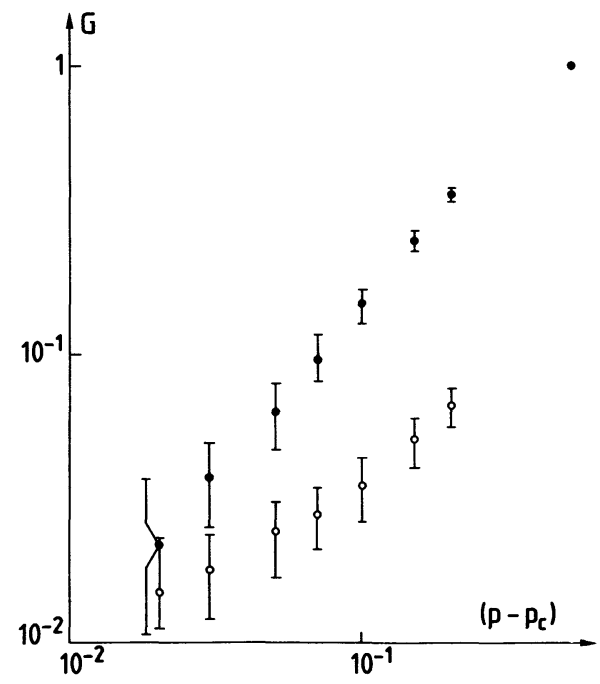

Fig. 5. - Double logarithmic plot of the conductivity of the network as a function of $p-p_{\mathrm{c}}$ for the $80 \times 80$ network. Shown is the initial conductivity, $G_{\text {in }}$, before any fuse is burned $(\bullet)$, and the final conductivity, $G_{\text {fin }}$, just before last fuse is burned (O). Statistics as in figure 3. 
Thus for a finite size system, $G_{\text {fin }}$ will vary as $\left(p-p_{\mathrm{c}}\right)^{v}$ until $\xi$ becomes larger than $L$, and subsequently, $G_{\text {fin }}$ and $G_{\text {in }}$ should be equal. Finally, it is worth noting that near $p_{\mathrm{c}}$ very few bonds need to burn in order to break the network. Therefore, geometrical exponents such as the fractal dimension of the initial and final network will be the same. On the other hand, the dynamical exponents such as the conductivity exponent or the fraction dimension are quite different [19]. This demonstrates the predominant role that the hottest bonds play in determining dynamic exponents.

Various properties of the interface produced by the breaking process, which separates the two disconnected portions of the network, also seem to be worth investigating. Unfortunately, several of the simpler properties that can be defined, such as the fractal dimension of the interface and the distance between successive bond cuts, exhibit very strong finite size dependence. In order to obtain reliable data on these quantities, we will need to consider system sizes much larger than what is currently possible within the framework of the present model.

Another property that might be of interest is the number of horizontal fuses burned divided by the number of vertical fuses burned. For $p=1$ one expects this ratio to be zero and for $p \rightarrow p_{\mathrm{c}}$ to be one. We note at this point that the nodes and links picture [16-17] cannot explain the conductivity exponent. Therefore we do not expect an effective medium theory to give very accurate results either.

In summary, we have introduced a new model that describes the breaking process of a random electrical network of insulators and fuses. This model is tractable numerically, and yields a number of interesting features that seem to be characteristic of the far from equilibrium properties that are typical of a breaking process. We have found that several characteristic quantities exhibit power-law dependences on $\left(p-p_{c}\right)$ with new exponents which are not apparently related to the known exponents of percolation. Further studies of random fuse and related models seem worthwhile, and work along these lines is currently in progress. It would also be very interesting to find experimental realizations for simple random fuse networks.

\section{Acknowledgments.}

We are extremely grateful to E. Guyon for many illuminating discussions.

\section{References}

[1] Kendall, K., in AIP Conference Proceedings No. 107 Physics and Chemistry of Porous Media (1983), and references therein.

[2] Smalley, R. F., Turcotte, D. L. and Solla, S. A., J. Geophys. Res. (1984).

[3] Englman, R., Jaeger, Z. and Levi, A., Philos. Mag. B 50 (1984) 307.

[4] GuR, Y., JAEGer, Z. and Englman, R., Eng. Fract. Mech. (1984).

[5] Ray, P. and Chakrabarti, B. K., Solid State Commun. 53 (1985) 477 ; Ray, P. and Chakrabarti, B. K., J. Phys. C (1985).

[6] See e.g., Fracture Vol. I-VII, H. Liebowitz (Ed.) (Academic Press, New York) 1984.

[7] LAWn, B. R. and WILShaw, T. R., Fracture of Brittle Solids (Cambridge University Press, U.K.) 1975.

[8] Zabolitsky, J. G., Phys. Rev. B 30 (1984) 4076.

[9] Herrmann, H. J., Derrida, B. and Vannimenus, J., Phys. Rev. B 30 (1984) 4079.

[10] Hong, D. C., Havlin, S., Herrmann, H. J. and Stanley, H. E., Phys. Rev. B 30 (1984) 4083.

[11] LobB, C. J. and Frank, D. J., Phys. Rev. B 30 (1984) 4089.

[12] Feng, S. and Sen, P., Phys. Rev. Lett. 52 (1984) 216.

[13] Kantor, Y. and Webman, I., Phys. Rev. Lett. 52 (1984) 1891.

[14] Bergmann, D. and Kantor, Y., Phys. Rev. Lett. 53 (1984) 511.

[15] Herrmann, H. J., Hong, D. C. and Stanley, J. Phys. A 17 (1984) L261.

[16] Skal, A. S. and Shklovskil, B. I., Sov. Phys. Semicond. 8 (1975) 1029.

[17] De GenNes, P. G., La Recherche 7 (1976) 919.

[18] Coniglio, A., J. Phys. A 15 (1982) 3829.

[19] See e.g., Dhar, D. and Ramaswamy, R., Phys. Rev. Lett. 54 (1985) 1346. 